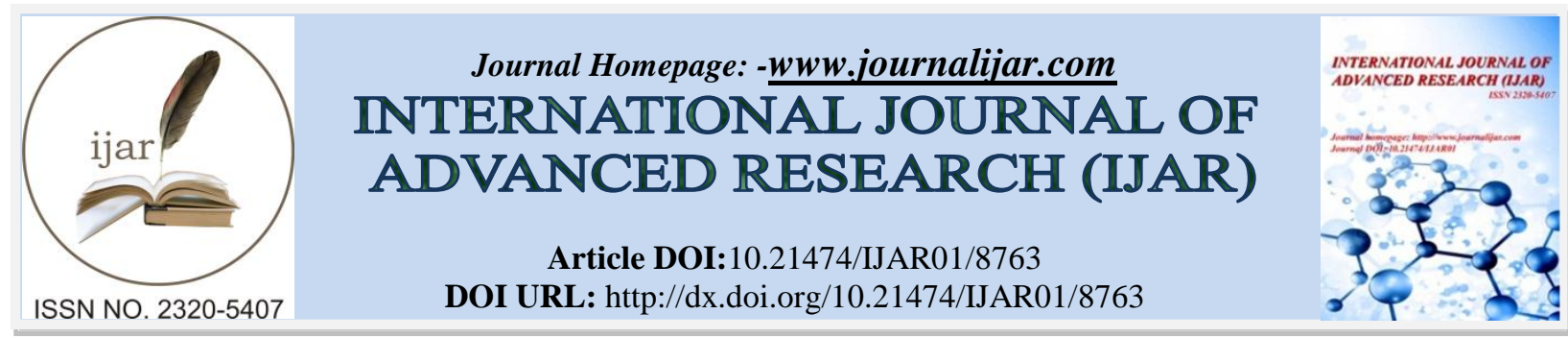

RESEARCH ARTICLE

\title{
A RANDOM PROJECTION APPROACH TO SECURE MEDICAL IMAGES.
}

Dr. A.Viji Amutha Mary.

Associate Professor, Dept of CSE, Sathyabama Institute of Science and Technology, Chennai-600119, India.

\section{Manuscript Info}

\section{Manuscript History}

Received: 20 January 2019

Final Accepted: 22 February 2019

Published: March 2019

Key words:-

Behaviour, determinant, factor, health, seeking.

\begin{abstract}
All our day's work in this world of science is done with useful data. These useful data or information is extracted from raw facts. From the information, knowledge is gained. This knowledge is used by the customers for beneficial outcome. Here comes the concept of data mining. An important query arises as how to preserve these data. This concept is called as Privacy Preserving with Data Mining (PPDM). Many PPDM techniques are available to protect the data. The PPDM technique is useful in fields like medicine, forensics, defence, etc to preserve the confidential data. The existing techniques protect the secret data either by perturbing or by hiding them.

Moreover, most of the techniques focus only on the numerical data. Very few perturbation techniques like translation, multiplicative and rotation perturb the images. But these techniques are very easily attacked by third parties since the transformation is a linear one. The security strength of these perturbation techniques is very low. Other perturbation techniques such as k- Anonymity, Rule Hiding and Data Swapping are applicable to numerical data. Excessive k-Anonymity also leads to data loss. Morphological operations greatly change the shape and structure of an image. But they are also reactive to noise and intrusions on the boundaries of the image. The privacy level of the images with these perturbation techniques is very low.

The Research Work aims to overcome the drawbacks of the existing perturbation techniques. The objective of the Research Work is to improve the privacy level of the images and to strengthen the security of images by implementing a Random Projection technique. The scope of the Research Work is confined to medical images. Medical images with various dimensions such as 2D, 3D and 5D are taken. Dimensionality Reduction plays a key role in this Research Work. Dimensionality of the input images is reduced by applying Random Projection technique.
\end{abstract}

Copy Right, IJAR, 2019,. All rights reserved.

\section{Introduction:-}

Geometric Data Perturbation Techniques

The following are the two widely used geometric data perturbation techniques.

Corresponding Author:-Dr. A.Viji Amutha Mary.

Address:-Associate Professor, Dept of CSE, Sathyabama Institute of Science and Technology,

Chennai-600119, India. 


\title{
Rotation perturbation
}

Turn annoyance was at first proposed for security saving information grouping. Pivot irritation is one of the real segments in geometric bother. Revolution irritation is essentially characterized as $G(X)=R X$ where $R$ is a haphazardly produced turn network and Xis the first information. The one of a kind advantage and furthermore the real shortcoming is separation protection, which is a bother invariant variable, yet causes remove surmising assaults. Separate induction assaults have been tended to by Chen and Liu (2007). Proposals were given to enhance its assault strength, which brings about the proposed geometric information bother. Other than turn bother, the scaling change is additionally utilized as a part of security safeguarding grouping. Scaling changes the separations, however the geometric choice limit is as yet kept up.

\section{Additive perturbation}

The added substance annoyance is a strategy for protection saving information mining in which clamor is added to the information so as to cover the quality estimations of records. The commotion included is adequately huge with the goal that individual record esteems can't be recuperated. Hence, methods are intended to get total dispersions from the irritated records. In this manner, information mining methods can be created keeping in mind the end goal to work with these total circulations. The essential technique is to camouflage the first esteems by infusing certain measure of added substance irregular commotion, while the particular data, for example, the segment dispersion, can in any case be adequately reproduced from the bothered information.

\section{Matrix Multiplicative Perturbation}

The most widely recognized technique for information bother is that of added substance irritations. Be that as it may, grid multiplicative irritations can likewise be utilized for protection safeguarding information mining. The first information framework is duplicated by clamor information to get a misshaped information grid. This annoyance around jam is Euclidean separations. Be that as it may, the first information can be effectively hacked.

\section{Random Projection (RP):}

The Random Projection concept arises from the theorem of Johnson Lindenstrauss. In Random Projection (RP), the original high-dimensional data is projected onto a lower-dimensional subspace using a random matrix whose columns have unit lengths. The dimensions and distribution of random projections matrices are controlled so as to preserve the pairwise distances between any two samples of the dataset. Thus random projection is a suitable approximation technique for distance based method. In general, RP technique is a tool for reducing the dimensionality of images.

\section{Need for Random Projection (RP) Technique:}

Since RP acts as a tool for reducing the image dimensions, the structure and shape of the image is greatly changed. This improves the privacy level of the images.

\author{
The benefits of RP technique are \\ It is simple. \\ It is computationally efficient. \\ It is less prone to errors \\ Processing takes place at a faster rate.
}

\section{Other salient features are}

1. RP Technique acts as an efficient dimensionality reduction tool for higher dimensional real world data sets.

2. It maintains the pair wise similarities between two data sets very well.

3. It is fruitful in applications in which there is a great influence in the distances of the actual space.

4. RP does not suffer from the impact of dimensionality.

Due to the salient features and advantages of RP compared to the other dimensionality reduction methods, RP is deployed in the research work.

\section{Radon Transform Technique}

The Radon transform is the integral transform which takes a function $f$ defined on the plane to a function $R f$ defined on the space of lines in the plane, whose value at a particular line is equal to the line integral of the function over 
that line. Random Projection is implemented by means of Radon transform technique. Radon further included formulas for the transform in three dimensions, in which the integral is taken over planes. It was later generalized to higher-dimensional Euclidean spaces, and more broadly in the context of integral geometry. The complex analog of the Radon transform is known as the Penrose transform. The Radon transform is widely applicable to tomography, the creation of an image from the projection data associated with cross-sectional scans of an object.

\section{Proposed Algorithm}

The step by step procedure of the 'PROJECTEDGE' technique is given below.

Algorithm:

Input: An image A (a, b) and a matrix generated randomly $C=(k \times a)$ where $k<a$.

The input image $\mathrm{A}$ is projected into a lower dimensional plane and the perturbed form is given by Equation 1

$$
X=\frac{1}{\sqrt{k \sigma_{c}}} C A
$$

Where $\sigma_{\mathrm{c}}$ is the variance

The resultant image after perturbation is $\mathrm{X}$.

The privacy level of the distorted image $Y$ is calculated by Equation (2).

$$
S=\frac{\operatorname{Var}(A-Y)}{\operatorname{Var}(A)}
$$

\section{Comparison with the existing techniques}

The privacy level of the RP technique is compared with the other existing techniques like translation, rotation and multiplicative perturbation and the result is depicted in Fig 1. The privacy level is calculated by finding the variance between the original image and the perturbed image. From Fig 1, it is observed that the privacy level of $\mathrm{RP}$ technique is higher than that of other existing techniques.

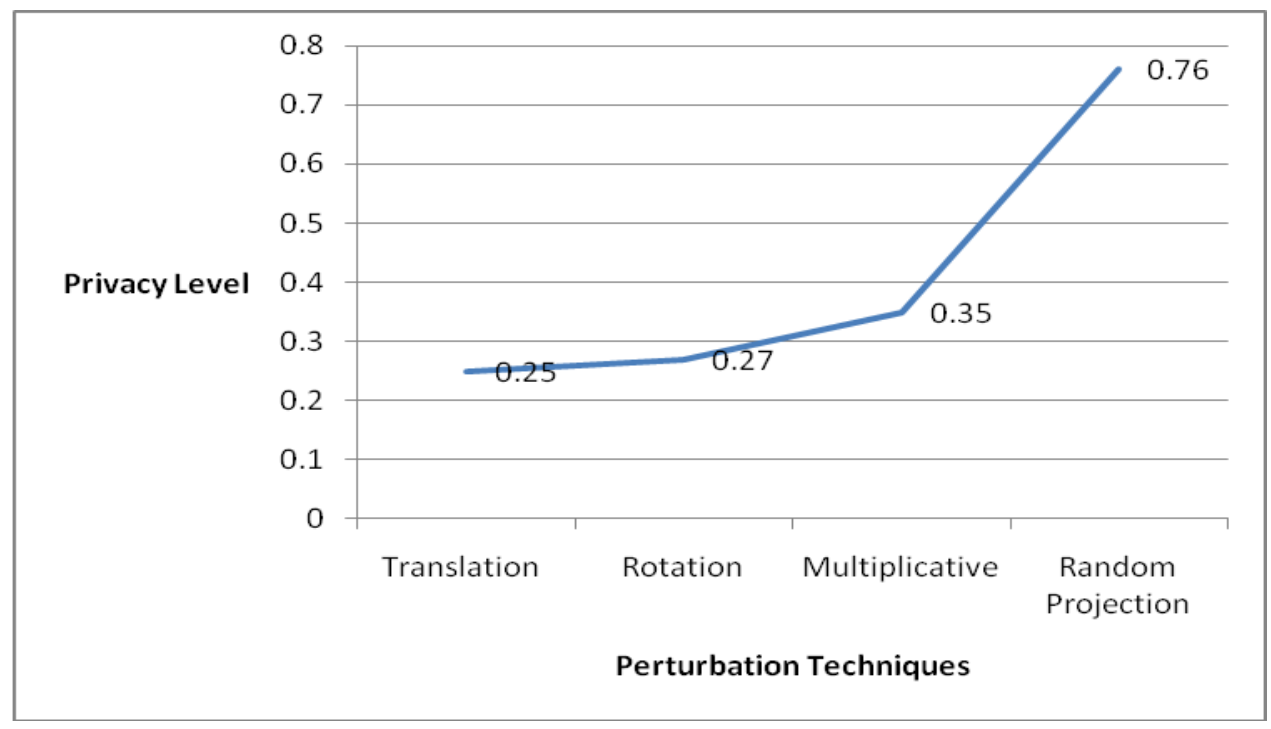

Fig 1:-Comparison of privacy level of RP technique with that of other existing techniques.

\section{Conclusion:-}

Since the RP technique greatly distorts the size and structure of the image, the privacy level of the image is high. The technique is compared with the existing perturbation techniques like translation, rotation, multiplicative. 
The privacy level of RP technique is higher compared to the other techniques. Thus the images can be highly secured with this approach. This approach can be combined with other distortion techniques to further improve the privacy level of images.

\section{References:-}

1. Dhivakar K. and, Mohana (2014), "A Survey on privacy preservation approaches and techniques", International Journal of Innovative Research in Computer and Communication Engineering Vol. 2, No.11.

2. Kaniska Bhaduri, Member IEEE, Mark d. Stefanski, and Ashok N. Shrivastava(2011), "Privacy-Preserving Outlier Detection through Random Nonlinear Data Distortion.” IEEE Transaction on System Man and Cybernetics Vol. 41, Issue 1.

3. Yaping Li, Minghua Chen, Qiwei Li and Wei Zhang (2012), "Enabling Multilevel Trust in Privacy Preserving Data Mining." IEEE Transactions on Knowledge and Data Engineering, Vol. 24, No. 9.

4. Viji Mary, Justin Samuel (2018), "A novel technique to secure the acute myocardial infarcta images by the enhancement of privacy level", Biomedical Research, Volume 29 (1), pp: 15-21.

5. Albert Mayan J , Kumar Akash Deep, Mukesh Kumar, Livingston Alvin , Siva Prasad Reddy (2016), "Number plate recognition using template comparison for various fonts in MATLAB ", IEEE International Conference on Computational Intelligence and Computing Research, ICCIC ,PP: 1-6 .

6. Naga Laxmi M. and Sandhya Rani K. (2013), "SVD based Data Transformation Methods for privacy preserving clustering", International Journal of Computer Applications, Vol.78, No.3.

7. Santosh Kumar Bhandare (2014), "Data transformation and encryption based privacy preserving Data mining System", International Journal of Advanced Research in Computer Science and Software Engineering, Vol. 4, No. 7.

8. Pui K Fong and Jens H. Webber-Jahnke (2012), "Privacy Preserving Decision Tree Learning Using Unrealized Data Sets" IEEE Transactions on Knowledge and Data Engineering Vol.24, No. 2.

9. Sweeney, L. (2002), "k-Anonymity A Model for Protecting Privacy", International Journal of Uncertainty, Fuzziness, and Knowledge-Based Systems, Vol.10, No. 5, pp. 557-570. 